\title{
Dolichoectatic aneurysms of the vertebrobasilar system: clinical and radiographic factors that predict poor outcomes
}

\author{
David S. Xu, MD, ${ }^{1}$ Michael R. Levitt, MD, ${ }^{2,3}$ M. Yashar S. Kalani, MD, PhD, ${ }^{1}$ \\ Leonardo Rangel-Castilla, MD, ${ }^{4}$ Celene B. Mulholland, MD, ${ }^{1}$ Isaac J. Abecassis, MD, ${ }^{2}$ \\ Ryan P. Morton, MD, ${ }^{2}$ John D. Nerva, MD, ${ }^{2}$ Adnan H. Siddiqui, MD, PhD, ${ }^{4}$ Elad I. Levy, MD, MBA, ${ }^{4}$ \\ Robert F. Spetzler, MD, ${ }^{1}$ Felipe C. Albuquerque, MD, ${ }^{1}$ and Cameron G. McDougall, MD'1
}

\begin{abstract}
'Department of Neurosurgery, Barrow Neurological Institute, St. Joseph's Hospital and Medical Center, Phoenix, Arizona; Departments of ${ }^{2}$ Neurological Surgery and ${ }^{3}$ Radiology, University of Washington, Seattle, Washington; and ${ }^{4}$ Department of Neurological Surgery, State University of New York at Buffalo, New York
\end{abstract}

\begin{abstract}
OBJECTIVE Fusiform dolichoectatic vertebrobasilar aneurysms are rare, challenging lesions. The natural history of these lesions and medium- and long-term patient outcomes are poorly understood. The authors sought to evaluate patient prognosis after diagnosis of fusiform dolichoectatic vertebrobasilar aneurysms and to identify clinical and radiographic predictors of neurological deterioration.

METHODS The authors reviewed multiple, prospectively maintained, single-provider databases at 3 large-volume cerebrovascular centers to obtain data on patients with unruptured, fusiform, basilar artery dolichoectatic aneurysms diagnosed between January 1, 2000, and January 1, 2015.
\end{abstract}

RESULTS A total of 50 patients (33 men, 17 women) were identified; mean clinical follow-up was 50.1 months and mean radiographic follow-up was 32.4 months. At last follow-up, $42 \%(n=21)$ of aneurysms had progressed and $44 \%(n=22)$ of patients had deterioration of their modified Rankin Scale scores. When patients were dichotomized into 2 groupsthose who worsened and those who did not-univariate analysis showed 5 variables to be statistically significantly different: $\operatorname{sex}(p=0.007)$, radiographic brainstem compression $(p=0.03)$, clinical posterior fossa compression $(p<0.001)$, aneurysmal growth on subsequent imaging $(p=0.001)$, and surgical therapy $(p=0.006)$. A binary logistic regression was then created to evaluate these variables. The only variable found to be a statistically significant predictor of clinical worsening was clinical symptoms of posterior fossa compression at presentation $(p=0.01)$.

CONCLUSIONS Fusiform dolichoectatic vertebrobasilar aneurysms carry a poor prognosis, with approximately onehalf of the patients deteriorating or experiencing progression of their aneurysm within 5 years. Despite being high risk, intervention-when carefully timed (before neurological decline)—may be beneficial in select patients.

https://thejns.org/doi/abs/10.3171/2016.10.JNS161041

KEY WORDS basilar artery; dolichoectasia; fusiform aneurysm; intracranial aneurysm; stroke; vascular disorders

$\mathrm{U}$ NRUPTURED fusiform vertebrobasilar artery aneurysms are rare heterogeneous lesions associated with significant morbidity and mortality. ${ }^{3,10,11,13,15}$ Little is known regarding their natural history, and even less guidance is available regarding management strategies in the modern era of microsurgical and endovascular treatment options. This multicenter retrospective study describes the medium- and long-term prognosis of patients with these lesions, and it identifies clinical and radiographic factors that predict neurological deterioration, which could aid in determining the timing for intervention.

\section{Methods}

Study Population

Using multiple, prospectively maintained, single-pro-

ABBREVIATIONS $\mathrm{CI}=$ confidence interval; $\mathrm{CN}=$ cranial nerve; $\mathrm{mRS}=$ modified Rankin Scale; OR = odds ratio; $\mathrm{SAH}=$ subarachnoid hemorrhage; $\mathrm{TIA}=$ transient ischemic attack. 
vider databases at Barrow Neurological Institute, the University of Washington, and the State University of New York at Buffalo, we queried all clinical reports for patients who had undergone diagnostic angiography between January 1,2000, and January 1, 2015, for the descriptive terms "fusiform" and "dolichoectatic." Subsequent radiological reviews of MR images, CT scans, and digital subtraction angiograms were then performed to identify patients harboring unruptured fusiform dolichoectatic aneurysms involving the basilar artery with or without continuity of disease into the vertebral arteries. Specific morphological features that were selected include the presence of fusiform segmental ectasia of the vessel lumen seen in Mizutani Type 2 aneurysms and the presence of an intraluminal or extraluminal thrombus with fragmentation or dissection of the intima consistent with Mizutani Type 3 aneurysms.? Only adult patients ( $\geq 18$ years old) with at least 4 weeks of clinical follow-up were included in the study. Patients who presented with acute neurological deterioration and were treated during the same hospitalization or within 30 days of diagnosis were excluded from the study to limit our analysis to factors affecting medium- and long-term natural history. All research workflow was conducted in accordance with an active hospital Institutional Review Board protocol.

\section{Data Collection}

Demographic data, including patient age, sex, family history, medical history, connective tissue disorders, anticoagulant and/or antiplatelet medications, and surgical or endovascular treatment, were recorded. Clinical symptomatology and neurological examination data were collected to assess the modified Rankin Scale (mRS) scores. On the basis of presenting signs and symptoms as well as imaging findings, the mechanism of symptoms was classified as being attributed to either direct compression of posterior fossa neural elements or ischemic events, such as stroke or transient ischemic attack (TIA).

\section{Statistical Analysis}

The primary end point was death or worsening of a patient's mRS score at the most recent clinical follow-up evaluation compared with the score at the time of diagnosis. For analysis, patients were dichotomized into 2 groups based on the primary outcome: those who had died or whose $\mathrm{mRS}$ scores had worsened, and those whose scores remained unchanged or improved. Univariate comparative analysis of demographic and clinical variables was made between the 2 groups using the chi-square test for discrete variables and the Mann-Whitney U-test for continuous variables.

Variables that were significant $(p<0.05)$ were then selected for further multivariate analysis. For this purpose, a binary logistic regression was constructed to query the predictive impact of multiple variables on the dichotomized primary outcome. Error due to the multiplicity of comparisons was minimized by applying the Bonferroni correction to the threshold for statistical significance, with a $\mathrm{p}$ value cutoff equal to $0.05 / \mathrm{m}$, where $\mathrm{m}$ is the number of independent variables evaluated. The quality of the logistic regression was assessed by applying the HosmerLemeshow goodness-of-fit test, and the regression model was compared with a constant-only model using the chisquare test. All statistical modeling was performed using SPSS Statistics for Windows software package (version 22.0, IBM Corp.).

\section{Results \\ Cohort Demographics}

A total of 50 patients were identified during the 15 -year study period: 26 from Barrow Neurological Institute, 13 from the University of Washington, and 11 from the State University of New York at Buffalo. A summary of clinical and demographic data is presented in Table 1. The mean age at diagnosis was 59.3 years (median 61.8 years, range 21.0-82.7 years). The mean clinical follow-up was 50.1 months (median 30.7 months, range 0.9-214.1 months), and the mean radiographic follow-up was 32.4 months (median 15.8 months, range 0-165.1 months). Sex distribution revealed a predominance of men $(33 ; 66 \%)$. Most patients $(44 ; 88 \%)$ had no family history of subarachnoid hemorrhage (SAH) and none had a history of a connective tissue disorder. All but 1 patient displayed neurological symptoms on diagnosis, with 27 (54\%) showing symptoms attributable to posterior fossa compression and $22(44 \%)$ showing evidence of stroke or TIA.

On radiographic studies, 43 aneurysms $(86 \%)$ had a maximum diameter $>7 \mathrm{~mm}$, and nearly half $(23 ; 46 \%)$ demonstrated signs of an intraluminal thrombus that caused brainstem compression. The mean maximum diameter of the basilar artery on admission was $15.8 \pm 8.85$ $\mathrm{mm}$. The $\mathrm{mRS}$ score distribution on presentation showed 42 patients $(84 \%)$ to be functionally independent with an $\mathrm{mRS}$ score $\leq 2$. During the course of imaging follow-up, 21 aneurysms $(42 \%)$ grew and $4(8 \%)$ ruptured, for an annualized risk of rupture rate of $1.9 \%$. As of the last clinical follow-up, 22 (44\%) of patients had worse mRS scores than at presentation; $16(32 \%)$ patients had died, with 11 of these deaths attributable to neurological deterioration. Causes of death were brainstem stroke in 10 patients (62\%); coronary artery disease in 3 patients (19\%); and sepsis, respiratory failure due to aspiration from possible brainstem dysfunction, and unknown cause in $1(6 \%)$ patient each.

\section{Univariate Analysis}

Patients were dichotomized on the basis of mRS score progression at last clinical follow-up (Table 2). Five variables were found to be statistically significantly different between these 2 groups: sex $(p=0.007)$, radiographic brainstem compression $(\mathrm{p}=0.03)$, clinical symptoms associated with brainstem compression ( $\mathrm{p}<0.001)$, aneurysmal growth on subsequent imaging $(\mathrm{p}=0.001)$, and surgical therapy ( $p=0.006$ ). The length of follow-up was not statistically different between the 2 groups.

\section{Multivariate Analysis}

We further analyzed the predictive impact of multiple statistically significant univariate variables on the likelihood of disease progression by creating a binary logistic 
TABLE 1. Summary of demographic data for 50 patients with dolichoectatic fusiform vertebrobasilar aneurysms

\begin{tabular}{|c|c|}
\hline Variable & Value $(\%)$ \\
\hline \multicolumn{2}{|l|}{ Continuous variables } \\
\hline \multicolumn{2}{|l|}{ Age (yrs) } \\
\hline Mean & 59.3 \\
\hline Median & 61.8 \\
\hline Range & $21.0-82.7$ \\
\hline \multicolumn{2}{|l|}{ Clinical follow-up (mos) } \\
\hline Mean & 50.1 \\
\hline Median & 30.7 \\
\hline Range & $0.9-214.1$ \\
\hline \multicolumn{2}{|l|}{ Radiographic follow-up (mos) } \\
\hline Mean & 32.4 \\
\hline Median & 15.8 \\
\hline Range & $0-165.1$ \\
\hline \multicolumn{2}{|l|}{ Categorical variables } \\
\hline \multicolumn{2}{|l|}{ Sex } \\
\hline Female & $17(34)$ \\
\hline Male & $33(66)$ \\
\hline \multicolumn{2}{|l|}{ Family history of SAH } \\
\hline No & $44(88)$ \\
\hline Yes & $6(12)$ \\
\hline \multicolumn{2}{|l|}{ mRS score on presentation } \\
\hline 0 & $3(6)$ \\
\hline 1 & $30(60)$ \\
\hline 2 & $9(18)$ \\
\hline 3 & $5(10)$ \\
\hline 4 & $2(4)$ \\
\hline 5 & $1(2)$ \\
\hline 6 & $0(0)$ \\
\hline Posterior fossa compression symptoms & $27(54)$ \\
\hline Stroke on presentation & $12(24)$ \\
\hline TIA on presentation & $10(20)$ \\
\hline \multicolumn{2}{|l|}{ Maximum aneurysm diameter } \\
\hline$\leq 7 \mathrm{~mm}$ & $7(14)$ \\
\hline$>7 \mathrm{~mm}$ & $43(86)$ \\
\hline Radiographic brainstem compression & $23(46)$ \\
\hline Thrombus present & $26(52)$ \\
\hline Antiplatelet therapy initiated & $37(74)$ \\
\hline Anticoagulant therapy initiated & $8(16)$ \\
\hline Endovascular therapy & $9(18)$ \\
\hline Surgical therapy & $15(30)$ \\
\hline Aneurysmal growth during imaging follow-up & $21(42)$ \\
\hline Aneurysmal rupture during clinical follow-up & $4(8)$ \\
\hline mRS score worsening during clinical follow-up & $22(44)$ \\
\hline Death during clinical follow-up & $16(32)$ \\
\hline
\end{tabular}

regression model and defining the dependent variable as whether a patient's mRS score had remained stable. The threshold for statistical significance was defined as $\mathrm{p}=$ 0.01 after applying the Bonferroni correction for the 5
TABLE 2. Demographic and clinical data according to $m R S$ score progression status at last follow-up visit

\begin{tabular}{|c|c|c|c|}
\hline \multirow[b]{2}{*}{ Variable } & \multicolumn{3}{|c|}{$\begin{array}{l}\text { mRS Score Progression } \\
\text { at Last Follow-Up }\end{array}$} \\
\hline & Yes (\%) & No $(\%)$ & $\mathrm{p}$ Value \\
\hline No. of patients & 22 & 28 & \\
\hline \multicolumn{4}{|l|}{ Continuous variables } \\
\hline Age (yrs) & & & 0.77 \\
\hline Mean & 60.4 & 58.4 & \\
\hline Median & 62.9 & 60.4 & \\
\hline Clinical follow-up (mos) & & & 0.12 \\
\hline Mean & 58.5 & 43.5 & \\
\hline Median & 44.4 & 24.1 & \\
\hline Radiographic follow-up (mos) & & & 0.78 \\
\hline Mean & 35.4 & 30.0 & \\
\hline Median & 15.8 & 15.8 & \\
\hline \multicolumn{4}{|l|}{ Categorical variables } \\
\hline Sex & & & 0.007 \\
\hline Male & $19(86.4)$ & $14(50.0)$ & \\
\hline Female & $3(13.6)$ & $14(50.0)$ & \\
\hline mRS score on presentation & & & 0.68 \\
\hline 0 & $2(9.1)$ & $1(3.6)$ & \\
\hline 1 & $14(63.6)$ & $16(57.1)$ & \\
\hline 2 & $4(18.2)$ & $5(17.9)$ & \\
\hline 3 & $2(9.1)$ & $3(10.7)$ & \\
\hline 4 & 0 & $2(7.1)$ & \\
\hline 5 & 0 & $1(3.6)$ & \\
\hline 6 & 0 & 0 & \\
\hline $\begin{array}{l}\text { Radiographic brainstem compres- } \\
\text { sion }\end{array}$ & $14(63.6)$ & $9(32.1)$ & 0.03 \\
\hline $\begin{array}{l}\text { Posterior fossa compression } \\
\text { symptoms }\end{array}$ & $19(86.4)$ & $8(28.6)$ & $<0.001$ \\
\hline Aneurysm diameter $\leq 7 \mathrm{~mm}$ & $2(9.1)$ & $5(17.9)$ & 0.38 \\
\hline Thrombus present & $14(63.6)$ & $12(42.9)$ & 0.14 \\
\hline $\begin{array}{l}\text { Aneurysmal growth during imaging } \\
\text { follow-up }\end{array}$ & $15(68.2)$ & $6(21.4)$ & 0.001 \\
\hline Antiplatelet therapy & $18(81.8)$ & $19(67.9)$ & 0.26 \\
\hline Anticoagulation therapy & $4(18.2)$ & $4(14.3)$ & 0.71 \\
\hline Endovascular therapy & $6(27.3)$ & $3(10.7)$ & 0.13 \\
\hline Surgical therapy & $11(50.0)$ & $4(14.3)$ & 0.006 \\
\hline
\end{tabular}

comparison variables. Within the model framework (Table 3), the only variable that was found to be a statistically significant predictor of mRS score progression was the presence of symptoms attributed to posterior fossa mass effect (odds ratio $[\mathrm{OR}]=0.057,95 \%$ confidence interval [CI] 0.006-0.504, $\mathrm{p}=0.01$ ).

The comparison of our regression model against a constant-only model showed all 5 variables to be statistically significant, indicating that they are distinguishers of $\mathrm{mRS}$ score progression $(\mathrm{p}<0.001)$. The Hosmer-Lemeshow goodness-of-fit test revealed that the regression was not statistically different from a null model without predictor variables, indicating a good fit $(\mathrm{p}=0.30)$. 
TABLE 3. Binary logistic regression for likelihood of stable $m R S$ score

\begin{tabular}{lccc}
\hline \multicolumn{1}{c}{ Variable } & OR & $95 \% \mathrm{Cl}$ & $\mathrm{p}$ Value \\
\hline Sex & 0.144 & $0.024-0.873$ & 0.04 \\
\hline Radiographic brainstem compression & 0.388 & $0.047-3.217$ & 0.38 \\
\hline $\begin{array}{l}\text { Posterior fossa compression symp- } \\
\quad \text { toms }\end{array}$ & 0.057 & $0.006-0.504$ & 0.01 \\
\hline Surgical therapy & 0.555 & $0.077-4.015$ & 0.56 \\
\hline Aneurysmal growth & 0.110 & $0.018-0.670$ & 0.02 \\
\hline
\end{tabular}

\section{Illustrative Cases}

\section{Case 1}

A 66-year-old man presented to an outside hospital with transient right-sided facial weakness. Imaging workup revealed a large, fusiform, basilar artery aneurysm with approximately $90 \%$ stenosis of the vessel lumen and a maximal diameter of $10.5 \mathrm{~mm}$. The patient was started on dual antiplatelet therapy with daily aspirin and clopidogrel, and he had no further neurological events until 12 years later when he presented to one of our institutions with recurrent facial weakness. At that time, follow-up angiography demonstrated complete thrombosis of the basilar artery (Fig. 1). The patient recovered to his neurological baseline after 3 weeks of neurorehabilitation and remained stable at the 1-year follow-up examination.

\section{Case 2}

A 58-year-old man presented with headaches and ataxia, and MRI revealed a fusiform basilar artery aneurysm. Angiography demonstrated a dolichoectatic lesion with a maximal diameter of $23 \mathrm{~mm}$ compressing the brainstem (Fig. 2A and B). Aspirin therapy (325 mg daily) was initiated. Four years later, the patient presented with worsening headaches and progressive lower-extremity weakness. Repeat angiography revealed progression of the lesion, with expansion of the maximal aneurysmal diameter to $32 \mathrm{~mm}$ (Fig. 2C and D). The patient underwent endovascular occlusion of the left vertebral artery and clip occlusion of the right vertebral artery distal to the origin of the posterior inferior cerebellar artery, resulting in elimination of flow along the proximal portion of the aneurysm (Fig. 2E). The patient was severely debilitated after intervention and was discharged to a skilled nursing facility. Fourteen months later, the patient had recovered to near baseline except for persistent paralysis of his left leg. Repeat CT angiography showed complete occlusion of the aneurysm (Fig. 2F).

\section{Case 3}

A 42-year-old woman presented with sudden-onset hemiplegia and palsy of the abducens cranial nerve (CN VI), the facial CN (CN VII), the glossopharyngeal CN (CN IX), and the hypoglossal CN (CN XII). Imaging workup demonstrated a large, partially thrombosed dolichoectatic basilar artery aneurysm with multifocal embolic strokes throughout the posterior fossa (Fig. 3A-C). Because of the presence of excellent collateral circulation from a primitive trigeminal artery, the patient was taken to
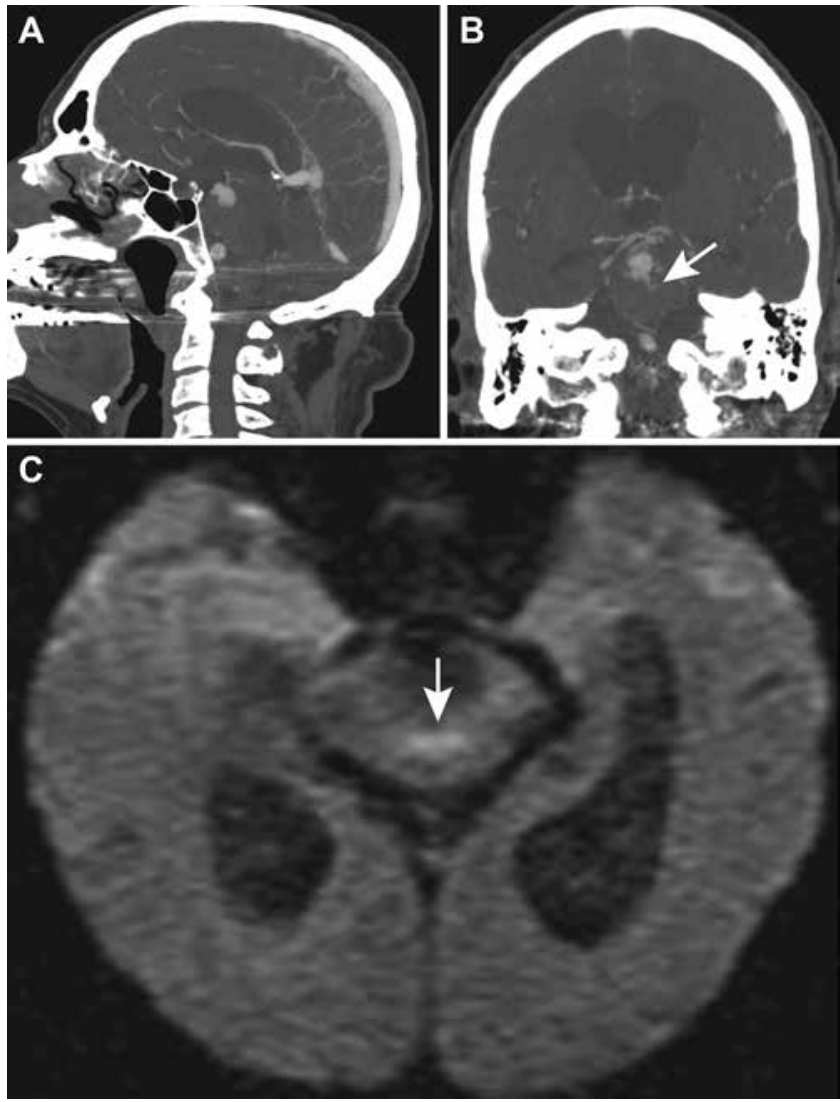

FIG. 1. Representative case of spontaneous aneurysm thrombosis. CT angiography with sagittal $(\mathbf{A})$ and coronal $(\mathbf{B})$ projections demonstrates complete thrombosis of the basilar artery, with intact flow channels supplying brainstem perforators (arrow). Diffusion-weighted MRI (C) demonstrates a small focus of diffusion restriction within the midpons (arrow).

the operative suite for clipping of the proximal basilar artery after its origin from the vertebral artery junction (Fig. $3 \mathrm{D})$. The patient was discharged with an mRS score of 5 and was dependent on a tracheostomy and a gastrostomy tube. After extensive rehabilitation, the patient was able to live independently and had an mRS score of 2 at the 1-year follow-up examination.

\section{Discussion}

Fusiform dolichoectatic basilar artery aneurysms are dangerous vascular entities that are believed to signify a poor prognosis. Steinberg et al. ${ }^{15}$ reviewed the natural history of large, complex posterior circulation aneurysms, including dolichoectatic lesions, within the accumulated case series by Drake and Peerless, ${ }^{3}$ and found that $80 \%$ of patients had died or were severely disabled by the 5 -year follow-up. Their data included patients who presented in a moribund state as well as multiple morphological subtypes of aneurysms, including giant saccular and junctional aneurysms. Since then, the natural history and treatment outcomes specific to dolichoectatic lesions have not been well defined because of their rarity. Most reports on fusiform vertebrobasilar aneurysms within the past 20 years are composed of small treated cohorts or a mixed population of different aneurysm subtypes and locations of origin. ${ }^{1}$ 


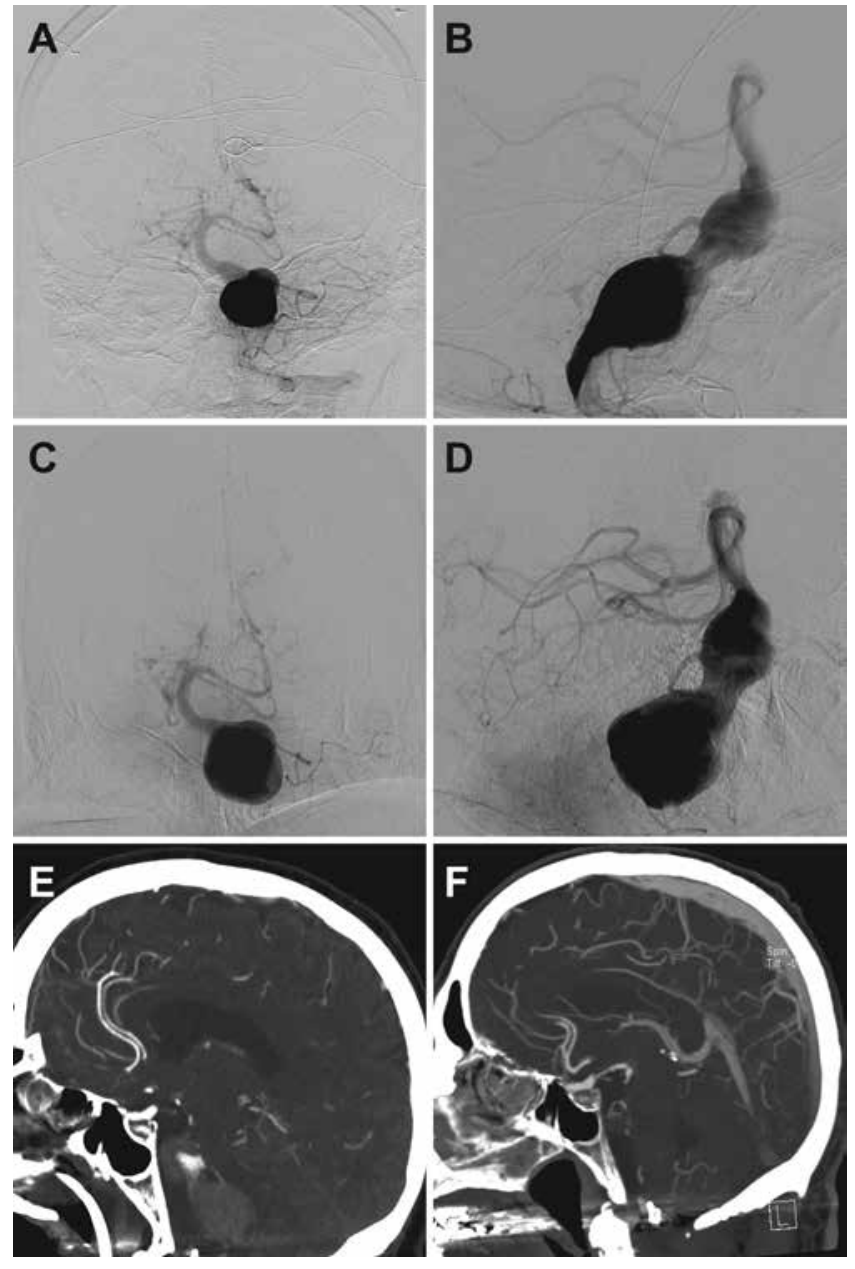

FIG. 2. Representative case of aneurysm progression. Digital subtraction angiography of a right vertebral artery injection on an anterior Townes projection $(\mathbf{A})$ and a lateral projection $(\mathbf{B})$ reveals a large fusiform dolichoectatic artery aneurysm encompassing the entirety of the vessel. Repeat digital subtraction angiography 4 years later shows interval progression of the aneurysm (C and D). Sagittal projections of CT angiography after flow reduction therapy show interval reduction of proximal aneurysm flow $(\mathbf{E})$ with subsequent complete occlusion of the aneurysm 14 months later (F).

A recent institutional review by Saliou et al. ${ }^{13}$ of a large tertiary referral center identified the incidence of nonacute dissecting fusiform basilar artery aneurysms to be only 33 $(1.3 \%)$ in 2522 patients. They found that these lesions averaged $10-15 \mathrm{~mm}$ in size and tended to be unstable, with radiographic progression occurring in most lesions and a rupture rate of $2(6.1 \%)$ in 33. Contrary to this report, a large study by Kobayashi et al. ${ }^{6}$ reviewed the cases of 113 patients with dissecting vertebrobasilar aneurysms and found the natural history of their lesions to be relatively benign. However, only 5 (4.4\%) of 113 presented with symptoms of brainstem mass effect, and none presented with stroke. They observed aneurysmal growth and new neurological symptoms from mass effect in 2 previously unaffected patients while a third patient with mass effect suffered a stroke; almost all patients $(110 ; 97.3 \%)$ were unchanged throughout the follow-up period. However, the mean aneurysm diameter of their cohort was 7-8 $\mathrm{mm}$.
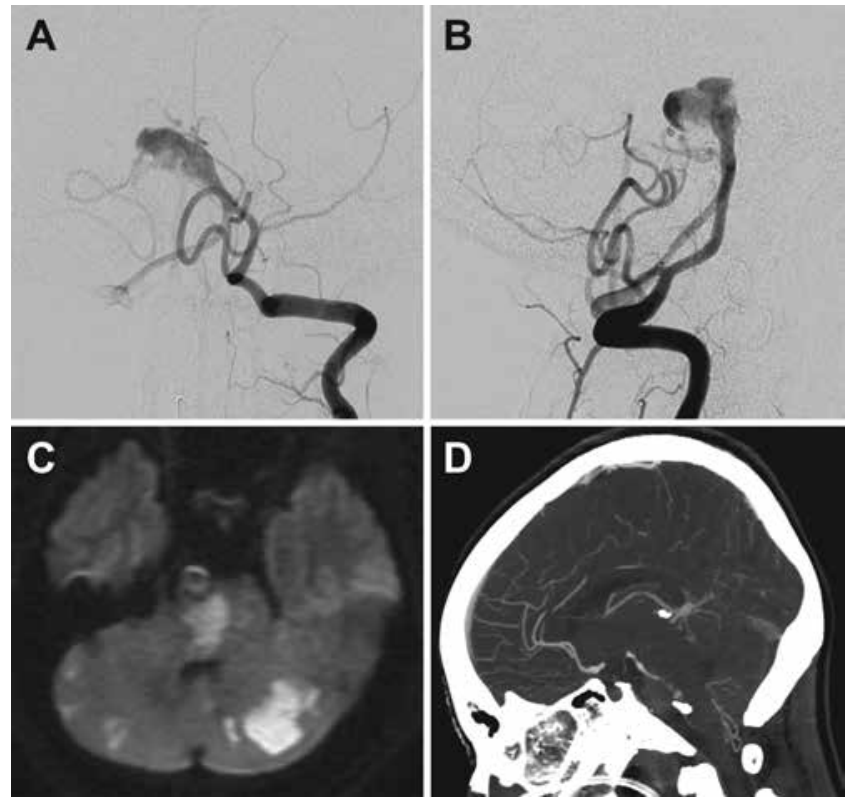

FIG. 3. Representative case of ischemic presentation of a partially thrombosed basilar artery aneurysm. Digital subtraction angiography of a left vertebral artery injection on an anterior Townes projection $(\mathbf{A})$ and a lateral projection (B) reveals a multilobulated fusiform basilar artery aneurysm encompassing the middle and distal segments of the basilar trunk. A diffusion-weighted MR image (C) shows multiple embolic strokes throughout the brainstem and cerebellum. A sagittal CT angiogram, taken after the patient underwent retrosigmoid craniotomy with clipping of the midbasilar artery proximal to the aneurysm, demonstrates occlusion of the aneurysmal sac (D), with patent retrograde flow through the distal basilar artery from posterior communicating artery collaterals.

Further analysis by Sacho et al. ${ }^{12}$ examined a cohort of 138 intradural aneurysms, including 29 that involved the basilar artery. Their study stratified aneurysms into groups comprising those that were atherosclerotic and those that were not, based on the review of 2 independent neuroradiologists. Fusiform aneurysms arising from atherosclerotic disease had a significantly higher risk of radiographic progression and patient mortality. Furthermore, aneurysms from both groups were more likely to progress if patients presented with neurological symptoms or if the maximal aneurysm diameter was larger than 7 $\mathrm{mm}$. We found similar results with regard to patient symptomatology, but did not find a 7-mm cutoff to significantly impact the risk of progression. Furthermore, we did not attempt to classify the hypothetical etiology of the aneurysm. Histological studies support the theory of dolichoectatic aneurysms as originating from intimal injury, with subsequent dissection of blood into the vessel media that over time results in dissolution of the elastic lamina and smooth muscle, leading to expansion and tortuosity of the vessel. ${ }^{9}$ Without histopathological analysis, any attempt to differentiate aneurysms into those that are incited and progress due to atherosclerotic disease and those that do not is highly subjective, given the overall high prevalence of atherosclerotic disease in our patients' demographics, and that, in practice, both aneurysm subtypes may behave similarly. 8,13

Our study provides insight into the demographics and 
natural history of this disease. As observed by others, there is a strong male predominance (2 to 1 ) and an average age of diagnosis in the fifties. ${ }^{4}$ Almost all our patients presented with signs or symptoms localized to the posterior fossa, and one-half demonstrated either discrete neurological deficits attributed to ischemic events or focal neurological findings attributable to mass effect on the brainstem or cerebellum. No patient had a history of connective tissue disorders, and only $6(12 \%)$ had a family history of SAH.

With regard to clinical course, approximately one-half (44\%) of the patients in our cohort experienced worsening of their mRS scores at a mean follow-up of 50.1 months, with death occurring in one-third of patients. Radiographic progression similarly occurred in $44 \%$ of patients within 3 years of follow-up. Interestingly, univariate analysis showed that multiple factors were associated with clinical decline, whereas multivariate analysis showed that only compressive symptomatology was a significant predictor of decline. This finding suggests that the long-term prognosis of patients depends heavily on their presenting condition. These results differ from the findings presented by Passero and Rossi, ${ }^{11}$ who evaluated the largest current series of patients with vertebrobasilar dolichoectasia, including several without aneurysmal dilation and only with increased vessel tortuosity. In their cohort of 156 consecutive patients, $48 \%$ experienced an ischemic or hemorrhagic stroke, and $43 \%$ of patients had radiographic progression of vessel disease at a mean follow-up of 11.7 years. Their analysis identified radiographic progression and initial basilar artery size as having the greatest impact on patient prognosis, but patient symptomatology on admission was not found to be a statistically significant determinant of future clinical events. In contrast to their study population, ours had more advanced disease, with an average basilar artery diameter on diagnosis nearly twice as large (15.8 $\mathrm{mm}$ vs $6.8 \mathrm{~mm}$ ), and the condition of our patients deteriorated more rapidly, with approximately the same incidence of clinical decline and radiographic progression but one-half the total duration of follow-up. Consequently, the acuity of clinical follow-up and discussion regarding intervention for patients with vertebrobasilar dolichoectatic aneurysms should not rely primarily on the radiographic appearance or progression of the lesion alone.

The prognostic implications from our results are important on multiple levels. The first is that the treatment of dolichoectatic aneurysms remains controversial and technically very challenging. Univariate and multivariate analyses showed that anticoagulant and antiplatelet treatment conferred no progression benefit, but given the overall high risk of ischemic events and the low incidence of aneurysmal rupture, the prophylactic use of both therapies is still reasonable. Both operative and endovascular treatment strategies have high rates of morbidity and mortality, $1,2,5,14,16$ and we cannot exclude the possibility that they may have contributed to the overall poor outcome of the cohort. Therefore, an understanding of the pathophysiology of these aneurysms and stringent patient selection are paramount before making any treatment decisions.

Figure 4 highlights the clinical pathways that these patients follow. Without intervention, the underlying vessel injury stimulates a mixed cycle of clotting and hemorrhage within the vessel wall, leading to 2 outcomes demonstrat-

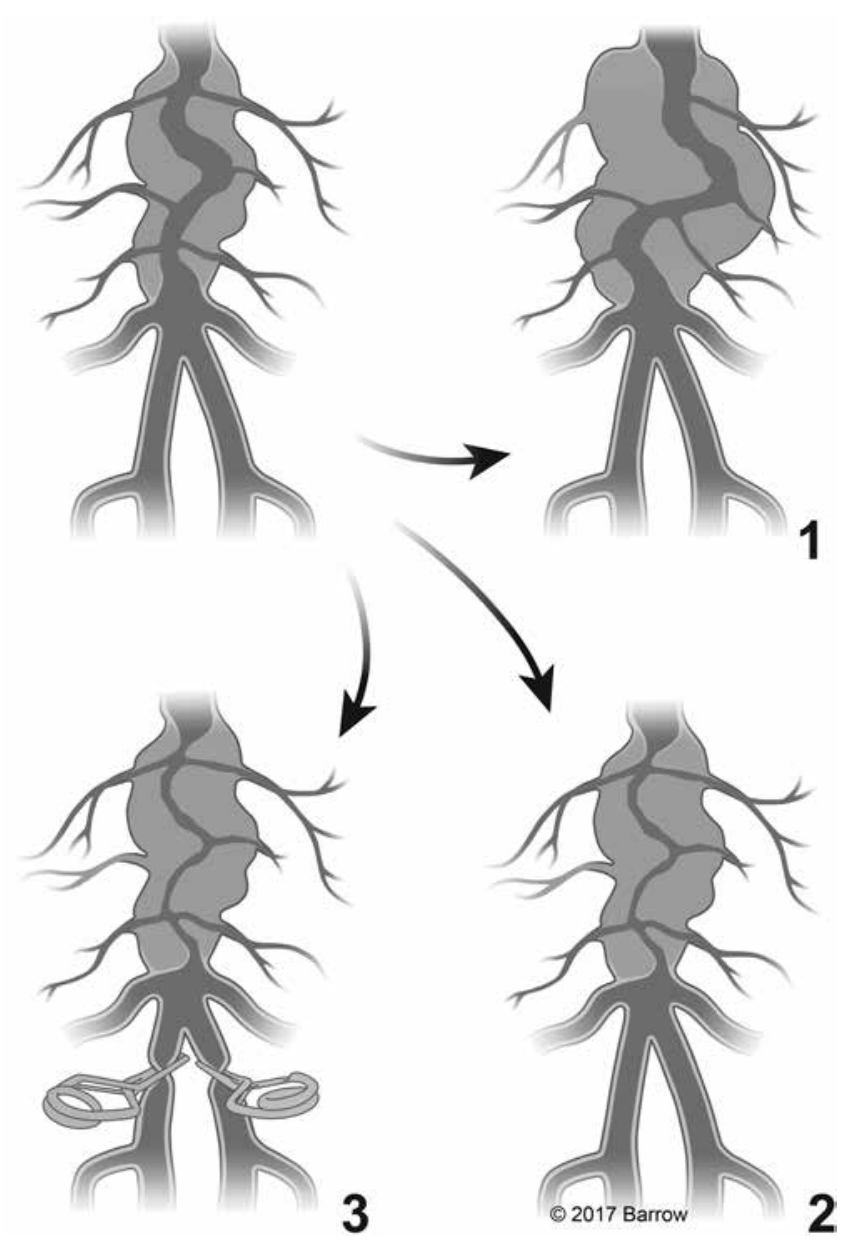

FIG. 4. Artist's depiction of clinical progression of fusiform dolichoectatic vertebrobasilar aneurysms. Without treatment, the aneurysm wall undergoes a repetitive cycle of hemorrhage and coagulation that may lead to (1) progression of disease along the vessel or (2) the aneurysm reaching a steady state, resulting in clotting of the parent vessel except for small channels of flow to perforating vessels. Treatment through flow reduction (3), by clipping either the bilateral vertebral arteries proximal to the posterior inferior cerebellar artery origin or the basilar artery proximal to the superior cerebellar artery origin in the perforator-free zone, attempts to push the pathological cycle toward clotting of the aneurysm with, ideally, preservation of perforators. Copyright Barrow Neurological Institute, Phoenix, Arizona. Published with permission.

ed by our case examples: 1) progression of the aneurysm leading to ischemic injury, hemorrhage, or worsening mass effect; or 2) spontaneous clotting of the aneurysmal vessel with or without patent flow to perforator territories. Given the morbidity associated with surgical or endovascular treatment and the small possibility that the aneurysm may spontaneously thrombose or stabilize over time, we recommend intervention only when compressive symptoms occur or when these aneurysms demonstrate radiographic progression. Selection of patients despite these findings remains challenging, but surgical or endovascular treatment may be suitable in unique circumstances, as observed in Cases 2 and 3.

There are multiple limitations to our study, such as its nonrandomized retrospective nature and selection bias, whereby asymptomatic patients with more benign aneu- 
rysms may be less likely to be referred to tertiary care centers participating in the study. Substantial conclusions cannot be drawn regarding the management of these rare lesions because of the relatively small number of patients in this cohort and the significant variation in their treatment. In addition, no patient was treated with flow-diverting stents, although this has been reported elsewhere with varying degrees of success. ${ }^{14}$ Furthermore, a detailed medical history regarding smoking and hypertension management was not available for each patient, and these are likely to be factors that impact the clinical courses of these patients. Ultimately, additional collaborative efforts such as a national patient registry may further help define the clinical prognosis and optimal treatment paradigms for patients with these challenging lesions.

\section{Conclusions}

Fusiform dolichoectatic basilar artery aneurysms are rare entities associated with a high risk of progression and morbidity. Clinical outcomes depend chiefly on the presence of symptoms of brainstem compression, and surgical or endovascular intervention remains a last resort for patients with symptomatic progression.

\section{References}

1. Anson JA, Lawton MT, Spetzler RF: Characteristics and surgical treatment of dolichoectatic and fusiform aneurysms. J Neurosurg 84:185-193, 1996

2. Coert BA, Chang SD, Do HM, Marks MP, Steinberg GK: Surgical and endovascular management of symptomatic posterior circulation fusiform aneurysms. J Neurosurg 106:855-865, 2007

3. Drake CG, Peerless SJ: Giant fusiform intracranial aneurysms: review of 120 patients treated surgically from 1965 to 1992. J Neurosurg 87:141-162, 1997

4. Echiverri HC, Rubino FA, Gupta SR, Gujrati M: Fusiform aneurysm of the vertebrobasilar arterial system. Stroke 20:1741-1747, 1989

5. Kalani MY, Zabramski JM, Nakaji P, Spetzler RF: Bypass and flow reduction for complex basilar and vertebrobasilar junction aneurysms. Neurosurgery 72:763-776, 2013

6. Kobayashi N, Murayama Y, Yuki I, Ishibashi T, Ebara M, Arakawa H, et al: Natural course of dissecting vertebrobasilar artery aneurysms without stroke. AJNR Am J Neuroradiol 35:1371-1375, 2014

7. Mizutani T, Miki Y, Kojima H, Suzuki H: Proposed classification of nonatherosclerotic cerebral fusiform and dissecting aneurysms. Neurosurgery 45:253-260, 1999

8. Nakatomi H, Segawa H, Kurata A, Shiokawa Y, Nagata K, Kamiyama H, et al: Clinicopathological study of intracranial fusiform and dolichoectatic aneurysms: insight on the mechanism of growth. Stroke 31:896-900, 2000

9. Nakayama Y, Tanaka A, Kumate S, Tomonaga M, Takebayashi S: Giant fusiform aneurysm of the basilar artery: consideration of its pathogenesis. Surg Neurol 51:140-145, 1999

10. Nishizaki T, Tamaki N, Takeda N, Shirakuni T, Kondoh T, Matsumoto S: Dolichoectatic basilar artery: a review of 23 cases. Stroke 17:1277-1281, 1986
11. Passero SG, Rossi S: Natural history of vertebrobasilar dolichoectasia. Neurology 70:66-72, 2008

12. Sacho RH, Saliou G, Kostynskyy A, Menezes R, Tymianski M, Krings T, et al: Natural history and outcome after treatment of unruptured intradural fusiform aneurysms. Stroke 45:3251-3256, 2014

13. Saliou G, Sacho RH, Power S, Kostynskyy A, Willinsky RA, Tymianski M, et al: Natural history and management of basilar trunk artery aneurysms. Stroke 46:948-953, 2015

14. Siddiqui AH, Abla AA, Kan P, Dumont TM, Jahshan S, Britz $\mathrm{GW}$, et al: Panacea or problem: flow diverters in the treatment of symptomatic large or giant fusiform vertebrobasilar aneurysms. J Neurosurg 116:1258-1266, 2012

15. Steinberg GK, Drake CG, Peerless SJ: Deliberate basilar or vertebral artery occlusion in the treatment of intracranial aneurysms. Immediate results and long-term outcome in 201 patients. J Neurosurg 79:161-173, 1993

16. van Oel LI, van Rooij WJ, Sluzewski M, Beute GN, Lohle PN, Peluso JP: Reconstructive endovascular treatment of fusiform and dissecting basilar trunk aneurysms with flow diverters, stents, and coils. AJNR Am J Neuroradiol 34:589-595, 2013

\section{Disclosures}

Dr. Siddiqui has direct stock ownership in StimSox, Valor Medical, Neuro Technology Investors, Cardinal Health, Medina Medical Systems, Buffalo Technology Partners Inc., and International Medical Distribution Partners; has served as a consultant to Codman \& Shurtleff Inc., Medtronic, GuidePoint Global Consulting, Penumbra, Stryker, MicroVention, W. L. Gore \& Associates, Three Rivers Medical Inc., Corindus Inc., Amnis Therapeutics Ltd., CereVasc LLC, Pulsar Vascular, The Stroke Project Inc., Cerebrotech Medical Systems Inc., Rapid Medical, Lazarus (acquired by Medtronic), Medina Medical (acquired by Medtronic), Reverse Medical (acquired by Medtronic), Covidien (acquired by Medtronic), Neuroavi, Silk Road Medical, and Rebound Medical; has board membership on the Intersocietal Accreditation Committee; and has served on National Principal Investigator/ Steering Committees for Penumbra, 3D Separator Trial, Covidien (now Medtronic), SWIFT PRIME and SWIFT DIRECT Trials (MicroVention), FRED Trial (MicroVention), CONFIDENCE Study, LARGE Trial, POSITIVE Trial (Penumbra), COMPASS Trial (Penumbra), and the INVEST Trial.

\section{Author Contributions}

Conception and design: Xu, Levitt. Acquisition of data: RangelCastilla, Mulholland, Abecassis, Morton, Siddiqui, Levy. Analysis and interpretation of data: Xu, Levitt. Drafting the article: Xu, Levitt, Kalani, Mulholland. Critically revising the article: McDougall, Xu, Levitt. Reviewed submitted version of manuscript: McDougall, Spetzler, Albuquerque. Statistical analysis: Xu, Levitt. Administrative/technical/material support: Nerva, Levy. Study supervision: McDougall, Siddiqui, Levy.

\section{Correspondence}

Cameron G. McDougall, c/o Neuroscience Publications, Barrow Neurological Institute, St. Joseph's Hospital and Medical Center, 350 W Thomas Rd., Phoenix, AZ 85013.email: neuropub@ dignityhealth.org. 